Research article

Open Access

\title{
Serum keratan sulfate transiently increases in the early stage of osteoarthritis during strenuous running of rats: protective effect of intraarticular hyaluronan injection
}

\author{
Tao Tang1, Takeshi Muneta1,2, Young-Jin Ju1 , Akimoto Nimura1 , Kyosuke Miyazaki \\ Hiroyuki Masuda ${ }^{3}$, Tomoyuki Mochizuki ${ }^{4}$ and Ichiro Sekiya ${ }^{4}$
}

\begin{abstract}
1Section of Orthopedic Surgery, Graduate School, Tokyo Medical and Dental University, 1-5-45 Yushima, Bunkyo-ku, Tokyo 113-8519, Japan ${ }^{2}$ Center of Excellence Program of Frontier Research on Molecular Destruction and Reconstruction of Tooth and Bone, Tokyo Medical and Dental University, 1-5-45 Yushima, Bunkyo-ku, Tokyo 113-8519, Japan

${ }^{3}$ Department of Pharmaceuticals Information, Seikagaku Corporation, 1-6-1 Marunouchi, Chiyoda-ku, Tokyo 113-8519, Japan

${ }^{4}$ Section of Cartilage Regeneration, Graduate School, Tokyo Medical and Dental University, 1-5-45 Yushima, Bunkyo-ku, Tokyo 113-8519, Japan

Corresponding author: Ichiro Sekiya, sekiya.orj@tmd.ac.jp
\end{abstract}

Received: 26 Nov 2007 Revisions requested: 21 Dec 2007 Revisions received: 16 Jan 2008 Accepted: 30 Jan 2008 Published: 30 Jan 2008

Arthritis Research \& Therapy 2008, 10:R13 (doi:10.1186/ar2363)

This article is online at: http://arthritis-research.com/content/10/1/R13

(c) 2008 Tang et al.; licensee BioMed Central Ltd.

This is an open access article distributed under the terms of the Creative Commons Attribution License (http://creativecommons.org/licenses/by/2.0), which permits unrestricted use, distribution, and reproduction in any medium, provided the original work is properly cited.

\begin{abstract}
Introduction Osteoarthritis is influenced by genetic and environment factors, including mechanical stress; however, the relationship between running and the development of osteoarthritis remains a matter of controversy. We investigated whether osteoarthritic change could be obtained in a rat strenuous running model, whether serum keratan sulfate in rats could be detected by HPLC and was associated with onset or progression of osteoarthritis, and whether hyaluronan injection suppressed development of osteoarthritis and elevation of serum keratan sulfate.
\end{abstract}

Methods Wistar rats were forced to run $30 \mathrm{~km}$ in 6 weeks on a treadmill machine. Articular cartilage of the knees was evaluated macroscopically and immunohistologically. Serum keratan sulfate was examined every week by HPLC. The effect of weekly knee injection of hyaluronan was also investigated.
Results Cartilage surfaces stained with India ink became irregular, metachromasia by safranin-O staining appeared to be almost lost, and Mankin's score significantly worsened after 30 $\mathrm{km}$ of running. Serum keratan sulfate in rats was detected by HPLC and transiently increased (peaked at 3 to 4 weeks) along with depletion of keratan sulfate in cartilage tissue. Hyaluronan treatment suppressed morphological progression of osteoarthritis and elevation of serum keratan sulfate.

Conclusion Rat strenuous running induced osteoarthritis. Serum keratan sulfate was associated with progression of osteoarthritis. Weekly intraarticular injection of hyaluronan controlled the development of osteoarthritis, and the effect was reflected by serum keratan sulfate.

\section{Introduction}

Osteoarthritis is the most common cause of joint pain and loss of mobility in older people. Osteoarthritis is influenced by genetic and environmental factors, including mechanical stress. To overcome difficulties in studying osteoarthritis in humans, animal models have been developed, such as spontaneous models in aging animals, genetically modified mice, as well as surgically, enzymatically or chemically induced models $[1,2]$. The use of strenuous running helps simulate long-term stress on weight-bearing joints. This model does not require surgical procedures or injection of reagents, and therefore it can detect subtle symptoms of osteoarthritis. The relationship between running and the development of osteoarthritis, however, remains a matter of controversy [3-7]. The first purpose of our study was to examine whether osteoarthritic change could be obtained in a rat strenuous running model.

Keratan sulfate is a glycosaminoglycan that is specifically distributed in the extracellular matrix of the cartilage, cornea, and brain [8]. Serum keratan sulfate was measured using an ELISA 
in 1985, and its usefulness as a marker of osteoarthritis was proposed; however, serum keratan sulfate did not correlate with the X-ray grading [9,10]. In 2007 Wakitani and colleagues measured serum keratan sulfate using HPLC, which has been reported to be more sensitive and more accurate than ELISA [11], and demonstrated a higher value of serum keratan sulfate in patients with early-stage damage of the articular cartilage undetectable by X-ray imaging [12]. These results indicate a more important usefulness of HPLC for serum keratan sulfate. On the other hand, recent analysis for serum keratan sulfate in rats and mice has scarcely been investigated possibly due to one paper describing the absence of keratan sulfate in skeletal tissues of mouse and rat [13]. In this paper, keratan sulfate was examined by immunohistochemistry using the monoclonal antibody MZ15; however, keratan sulfate expression in rat cartilage was detected using the other antibodies 5D4 [14] and EFG-11 [6]. The second study purpose was to investigate serum keratan sulfate in rats by HPLC and its association with onset or progression of osteoarthritis.

Hyaluronan is also a glycosaminoglycan. In articular cartilage, hyaluronan and aggrecan form large aggregates, bind huge amounts of water, and are responsible for the resilience of cartilage. Intraarticular injection of hyaluronan has been wildly utilized clinically as pain relief for the early stage of knee osteoarthritis $[15,16]$. Several studies have reported that hyaluronan has beneficial effects on cartilage during development of osteoarthritis [17]. The third study objective was to analyze serum keratan sulfate sequentially after hyaluronan injection in a rat strenuous running model.

\section{Materials and methods} Animals and strenuous running

Wistar rats 16 to 18 weeks of age (Sankyo Labo Service, Tokyo, Japan) were used for the experiments. All experiments were conducted in accordance with the institutional guidelines for the care and use of experimental animals. Rats were divided into three groups: no running group ( $0 \mathrm{~km}, n=5)$; only strenuous running group ( $30 \mathrm{~km}, n=8$ ); and strenuous running and hyaluronan injection group (15 km, $n=3 ; 30 \mathrm{~km}, n=$ $5)$.

For strenuous running exercise, a rodent treadmill machine (MK-680R5; ME Service., Tokyo, Japan) was used with a 5\% incline (Figure 1a). The MK-680R5 has been designed to compulsively make animals exercise by electrical shock delivered to the animals without failure by the adoption of a shock generator scrambler. The rats were acclimated to the treadmill by gradually increasing the running speed and time as follows: day 1,10 minutes at $10 \mathrm{~m} / \mathrm{min}$; day 2,15 minutes at $12 \mathrm{~m} / \mathrm{min}$; day 3,20 minutes at $15 \mathrm{~m} / \mathrm{min}$; day 4,30 minutes at $18 \mathrm{~m} / \mathrm{min}$; and day 5,35 minutes at $20 \mathrm{~m} / \mathrm{min}$. On day 8 and thereafter, the rats were forced to run for 55 minutes a day at $20 \mathrm{~m} / \mathrm{min}$, with the first 10 minutes consisting of a $12 \mathrm{~m} / \mathrm{min}$ warm-up. Rats ran $30 \mathrm{~km}$ in 6 weeks, as shown in Figure $1 \mathrm{~b}[18]$. For the hyaluronan injection group $(n=5), 100 \mu$ l hyaluronan (average molecular weight $=8 \times 10^{5} \mathrm{Da}$; Seikagaku Corp., Tokyo, Japan) containing $1 \mathrm{mg}$ in formulated concentrate was injected into the right knee. The injections were performed initially at 5 days and every 1 week thereafter under anesthesia of $10 \mathrm{mg}$ sodium pentobarbital (Dainippon Sumitomo Pharma, Osaka, Japan) by intraperitoneal injections. As a control for hyaluronan treatment, saline or PBS was not injected into the left knee to avoid the possibility that they might enhance osteoarthritis $[19,20]$.

\section{Macroscopic observation}

Femoral and tibial condyles were carefully dissected separately without damaging the cartilage surface, and were then stained with India ink to identify the location, size and severity of cartilage degeneration. Macroscopic pictures were taken

\section{Figure 1}

(a)

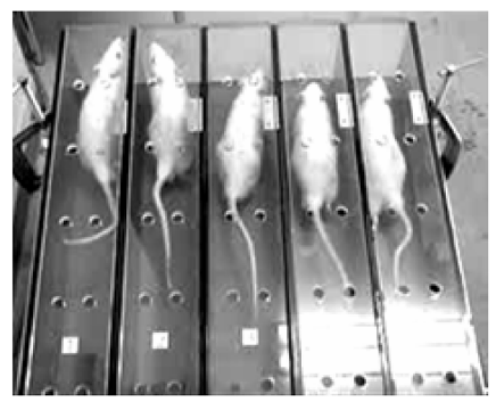

MK-680R5 (b) $(\mathrm{km})$

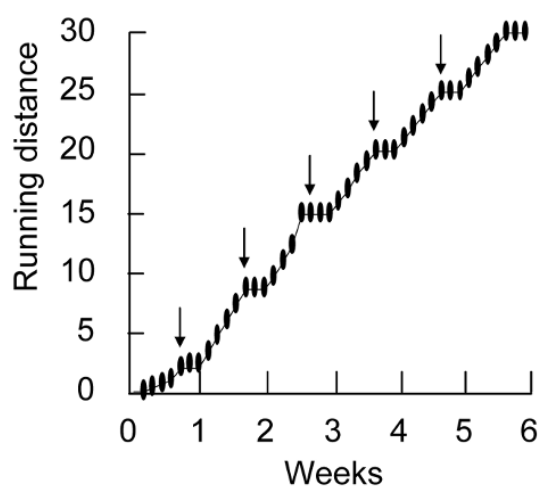

Method for rat strenuous running. (a) Rodent treadmill machine designed to compulsively make rats run. (b) Protocol for running exercise. Rats were forced to run $30 \mathrm{~km}$ in 6 weeks. For the hyaluronan group, the injection was performed initially at 5 days, followed by every 7 days (arrows). 
using a specification MPS-7 (Sugiura Laboratory Inc., Tokyo, Japan), a dedicated medical photography platform. Digital images were taken using a Nikon Coolpix 4500 digital camera (Nikon, Tokyo, Japan).

\section{Histology}

The rats were sacrificed with an overdose of sodium pentobarbital. Both femurs and tibias were fixed in 4\% paraformaldehyde at $\mathrm{pH} 7.4$ for 3 days, were decalcified in $20 \%$ ethylenediamine tetraacetic acid solution for 21 days, and were then embedded in paraffin wax. Femurs and tibias were sectioned sagittally at $5 \mu \mathrm{m}$ and stained with safranin-O. Histological sections were visualized using an Olympus IX71 microscope (Olympus, Tokyo, Japan). Each section was evaluated with the Mankin's histological grading system (Mankin's score: 0 to 14) for articular cartilage degeneration [21].

\section{Immunohistochemistry}

Sections were deparaffinized, washed in PBS, and pretreated with $0.4 \mathrm{mg} / \mathrm{ml}$ proteinase $\mathrm{K}$ (DAKO, Carpinteria, CA, USA) in Tris- $\mathrm{HCl}$ buffer for 15 minutes at room temperature for optimal antigen retrieval. Endogenous peroxidases were quenched using $0.3 \%$ hydrogen peroxide in methanol for 20 minutes at room temperature. The sections were rinsed once in PBS for 5 minutes and were briefly blocked with 10\% normal horse serum (Vector Laboratories, Burlingame, CA, USA) to avoid nonspecific binding of the antibody. The sections were then incubated in monoclonal anti-keratan sulfate antibody (5-D-4, 1:100 dilution with PBS containing 1\% BSA; Seikagaku Corp.) or anti-mouse monoclonal antibody against human type II collagen (1:200 dilution with PBS containing 1\% BSA; Daiichi Fine Chemical, Toyama, Japan) at room temperature for 60 minutes. After rinsing in PBS, the tissues were incubated with biotinylated horse anti-mouse IgG secondary antibody (Vector Laboratories) for 30 minutes at room temperature. The slides were again immersed in PBS and were incubated for another 30 minutes with Vectastain $A B C$ reagent (Vector Laboratories). Finally, the sections were shortly counterstained with hematoxylin, dehydrated and mounted in a xylol-soluble mount (Vitro-Clud; R. Langenbrinck, Emmendingen, Germany) $[12,22,23]$.

\section{Keratan sulfate concentration}

To avoid circadian variation of keratan sulfate in serum, blood was collected at between 3:00 and 4:00 pm, 1 hour after finishing strenuous running. Approximately $500 \mu$ blood was aspirated with a 27-gauge needle from the tail vein of the rats. The blood was kept at $4^{\circ} \mathrm{C}$ for 2 hours and was centrifuged at $2,000 \mathrm{rpm}$ for 15 minutes at $4^{\circ} \mathrm{C}$. The serum was separated, allocated into $100 \mu \mathrm{l}$, and kept frozen at $-70^{\circ} \mathrm{C}$. To avoid an influence of freeze-thaw, the serum was used for the analyses without refreezing. Every rat started strenuous running on Monday and had its blood aspirated every Friday. Each $200 \mu \mathrm{l}$ serum was diluted with $800 \mu$ distilled water, digested with $100 \mu \mathrm{l}$ of $2.0 \%$ Actinase E (Kaken Pharmaceutical., Tokyo,
Japan) at $55^{\circ} \mathrm{C}$ for 24 hours, and was heated at $100^{\circ} \mathrm{C}$ for 10 minutes. The whole solution was applied to $\mathrm{Q}$ Sepharose 0.15 M sodium chloride (GE Healthcare UK Ltd., Little Chalfont, Buckinghamshire, UK), and was extracted with $50 \mathrm{mM}$ Tris$\mathrm{HCl}$ buffer ( $\mathrm{pH}$ 8.6) containing $2 \mathrm{M}$ sodium chloride. The extracted material was desalinated with PD-10 (GE Healthcare), dried, and dissolved again by $0.2 \mathrm{ml}$ distilled water. Then $1 \mathrm{mU}$ Keratanase II (Seikagaku Corp.) was added, followed by addition of $40 \mu \mathrm{l}$ of $100 \mathrm{mM}$ sodium acetate buffer (pH 6.0), and the mixture was incubated at $37^{\circ} \mathrm{C}$ for 3 hours.

The sample was ultrafiltered using an Ultrafree C3GC system whose molecular weight cutoff was 10,000 daltons (Japan Millipore, Tokyo, Japan). The filtrate, which contained monosulfate disaccharide and disulfate disaccharide derived from keratan sulfate, was analyzed by HPLC with the column packed with polyamine-bound silica (YMC gel PA-120; YMC Ltd, Kyoto, Japan). The monosulfate disaccharide and disulfate disaccharide were eluted with a gradient of 0 to $100 \mathrm{mM}$ sodium sulfate for 45 minutes at a flow rate of $0.5 \mathrm{ml} / \mathrm{min}$. To elute from the column, $100 \mathrm{mM}$ sodium tetraborate buffer $(\mathrm{pH}$ 9.0) containing 1\% 2-cyanoacetamide was added at a flow rate of $0.5 \mathrm{ml} / \mathrm{min}$. The mixture was passed through poly(ether ether ketone) (PEEK; Victrex, Lancashire, UK) tubing with a $0.5 \mathrm{~mm}$ diameter and a $10 \mathrm{~m}$ length in a dry fluoromonitor (excitation, $331 \mathrm{~nm}$; emission, $383 \mathrm{~nm}$ ). The area of each peak corresponding to monosulfate disaccharide and to disulfate disaccharide was calculated by Borwin-HSS2000 software (Jasco, IJsselstein, Netherlands) and was converted to the amount of the corresponding disaccharides against the area of standard monosulfate disaccharide and disulfate disaccharide (Seikagaku Corp.) [12].

\section{Statistical analysis}

The StatView 5.0 program (SAS Institute, Cary, NC, USA) was used for statistical analyses. $P<0.05$ was considered statistically significant.

\section{Results}

\section{Degeneration of articular cartilage}

Strenuous running exercise affected the articular cartilage. The cartilage surfaces of both the lateral femoral condyle and the lateral tibial plateau were irregular after $30 \mathrm{~km}$ of strenuous running (Figure 2). A weekly intraarticular hyaluronan injection helped maintain the smoothness of the surface of the articular cartilage (Figure 2). Histological analyses demonstrated that $30 \mathrm{~km}$ of strenuous running induced depletion of the articular cartilage matrix (Figure 3a) and worsened the Mankin's score (Figure 3b). Hyaluronan treatment suppressed the degeneration of the articular cartilage (Figure 3c).

\section{Serum concentration of keratan sulfate}

Sequential serum concentrations of keratan sulfate were examined. In the control group (the only strenuous running group), the concentration transiently peaked at 3 to 4 weeks 

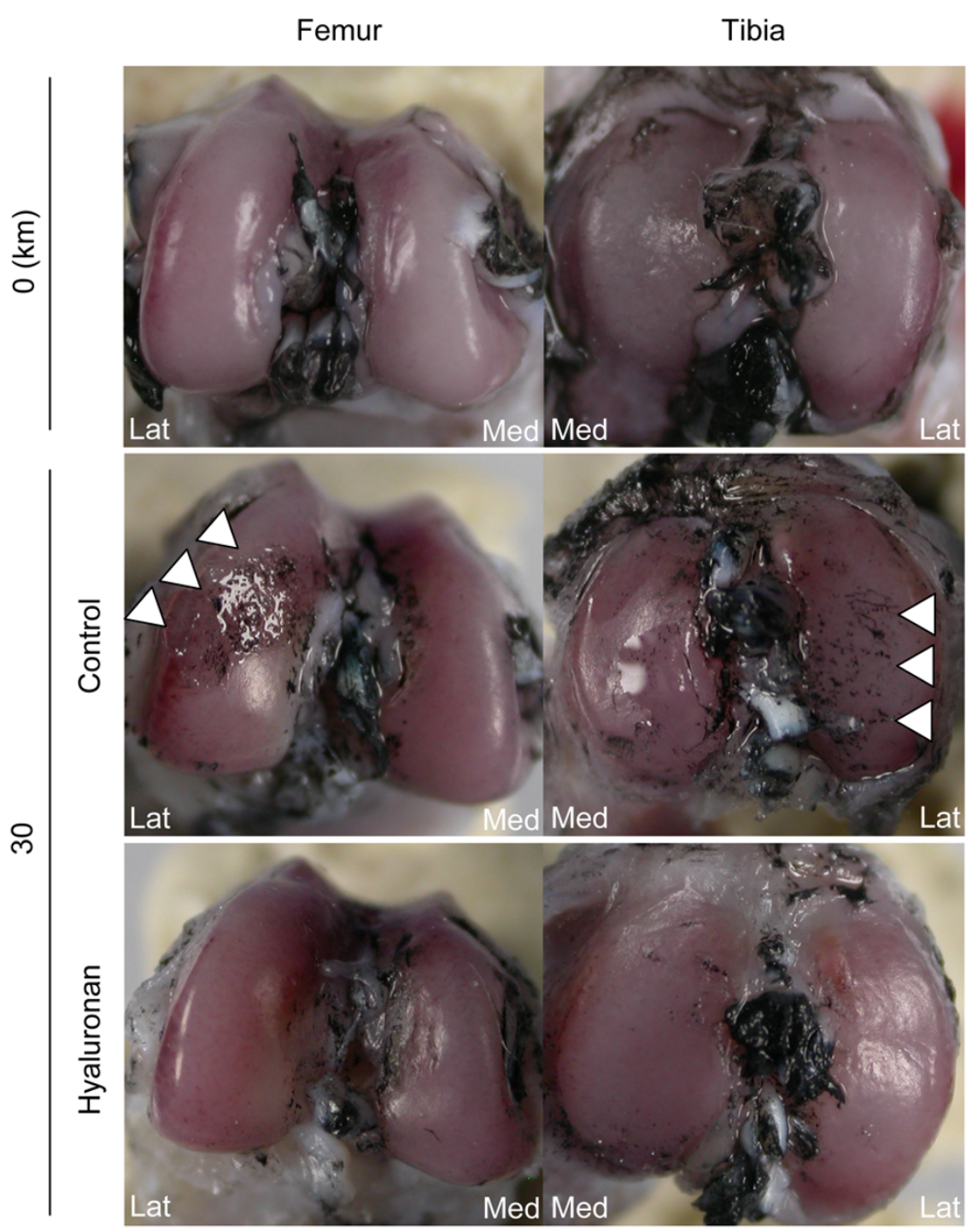

Macroscopic observation. Femoral and tibial articular cartilage stained with India ink. Cartilage lesions are indicated by arrowheads. Lat, lateral; Med, medial.

(Figure 4, left). Hyaluronan treatment appeared to suppress the keratan sulfate concentration (Figure 4, center). The average of the maximum keratan sulfate concentration during the 5 -week period in each rat in the hyaluronan group was significantly lower than that in the control group (Figure 4, right).

\section{Immunohistochemical analysis}

Keratan sulfate and type II collagen were expressed in cartilage of rats before strenuous running and were still present at 3 weeks after $15 \mathrm{~km}$ of strenuous running. Keratan sulfate expression decreased and was hardly observed at 6 weeks after $30 \mathrm{~km}$ of strenuous running in the control group. The type II collagen-positive area decreased along with the cartilage area, but the expression could be still observed in the remaining cartilage in the control group after $30 \mathrm{~km}$ of strenuous run- ning. Hyaluronan treatment suppressed the loss of keratan sulfate and type II collagen after $30 \mathrm{~km}$ of strenuous running (Figure 5).

\section{Discussion}

Running exercise may injure articular cartilage - although there are also studies suggesting that it has no adverse effects on articular cartilage, and that the effects are mostly beneficial [5]. These studies indicate that the influences of physical exercise are bidirectional, the net result dependent on the degree of joint loading. Excessive running seems to lead to a higher incidence of osteoarthritis, whereas moderate running is either noncontributory in joint degeneration or beneficial in decreasing the risk of osteoarthritis in animals. Excessive running load is expected to markedly exceed the animals' normal 


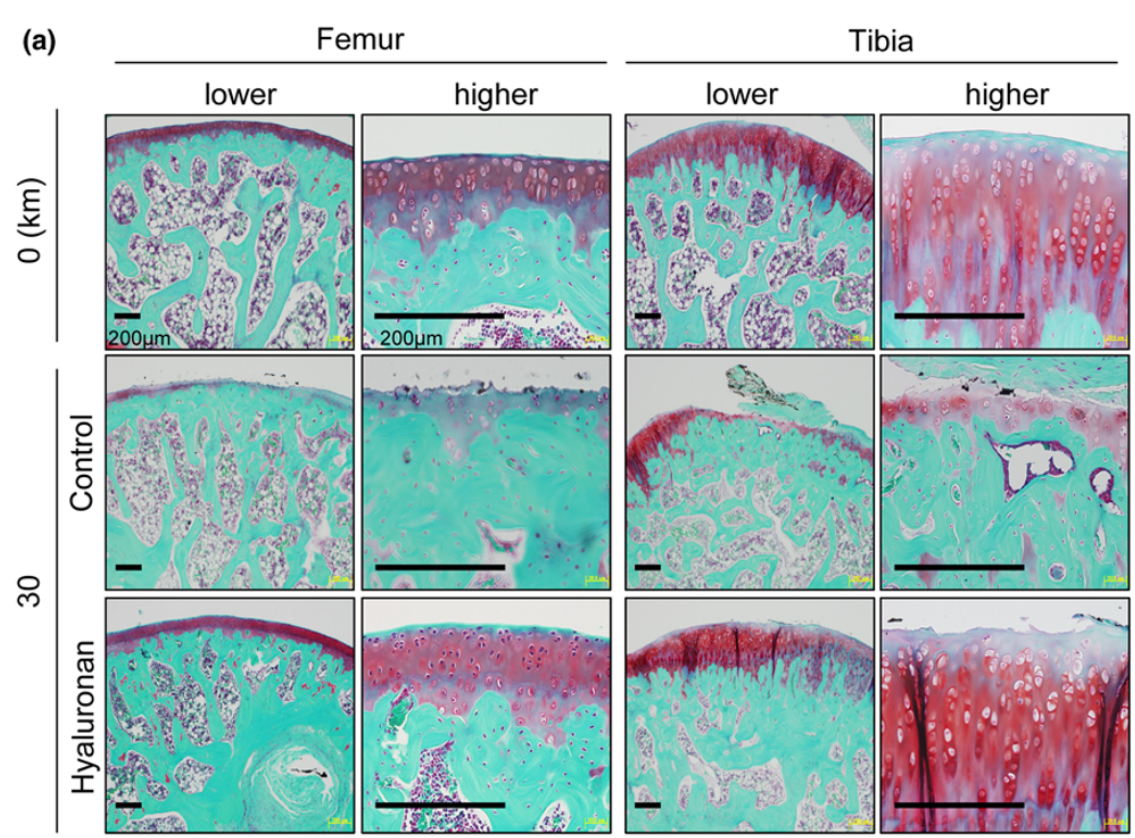

(b)

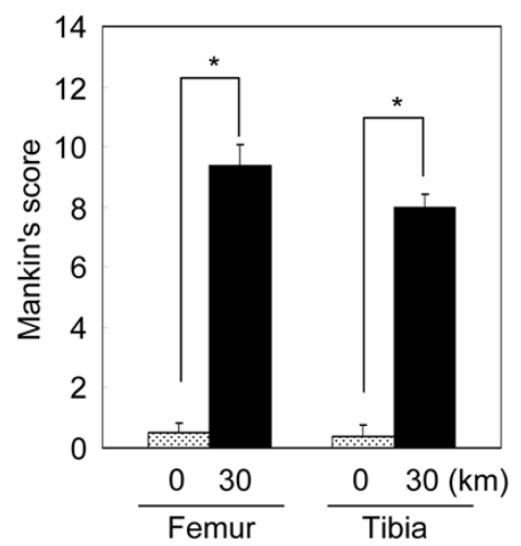

(c)

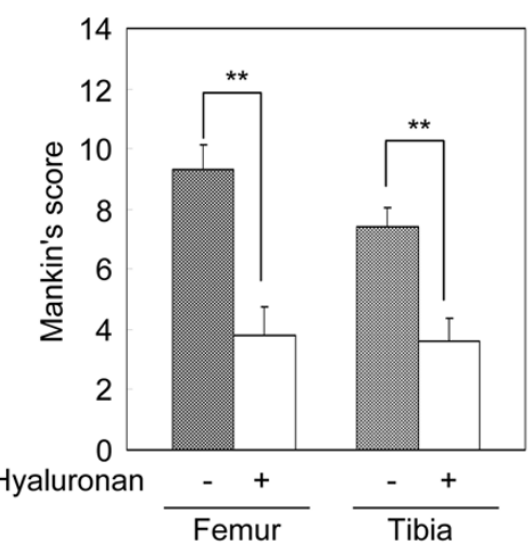

Histological analyses for cartilage legions. (a) Representative histologies of the lateral femoral condyle and the lateral tibial plateau stained with safranin-O. Scale bar $=200 \mu \mathrm{m}$. (b) Influence of running $30 \mathrm{~km}$ on cartilage. Mankin's score of the right knee in rats after $30 \mathrm{~km}$ of strenuous running without hyaluronan injection was compared with that in control rats without strenuous running or hyaluronan injection. Data expressed as the mean \pm standard deviation $(n=5)$. * $P<0.01$, Mann-Whitney $U$ test. (c) Effect of hyaluronan on cartilage. Mankin's score for cartilage legions. Rats were forced to run $30 \mathrm{~km}$ in 6 weeks. Hyaluronan was injected each week into the right knee. Nothing was injected into the left knee. Both sides of the knees were compared. Mankin's score expressed as the mean \pm standard deviation $(n=5)$. ${ }^{\star \star} P<0.05$, Wilcoxon signed rank test.

physiological running activities. Pap and colleagues first reported development of osteoarthritis in the knee joints of rats after strenuous running exercise [3]. They stimulated the rats intracranially to motivate them to run on a running wheel. In our present study, we used a rodent treadmill machine to motivate Wistar rats running by external electrical stimulation to their tail. We demonstrated development of osteoarthritis of the knee joint of nongenetically modified rats. This simple method without requiring specific surgery will be useful for analyses of subtle symptoms such as serum cartilage marker in the present study.
Progression of osteoarthritis is likely to result primarily from an imbalance between cartilage degradation and repair. Biological markers in the blood might provide relevant information more rapidly than imaging techniques such as radiography and magnetic resonance imaging can, and should contribute to our understanding of mechanisms that underlie the clinical efficacy of osteoarthritis treatments [24]. As osteoarthritis affects mainly the cartilage, structural molecules or fragments derived from cartilage tissues could be candidate serum cartilage markers for osteoarthritis. These tissues might also include molecules that play a role in metabolic processes, 
Figure 4

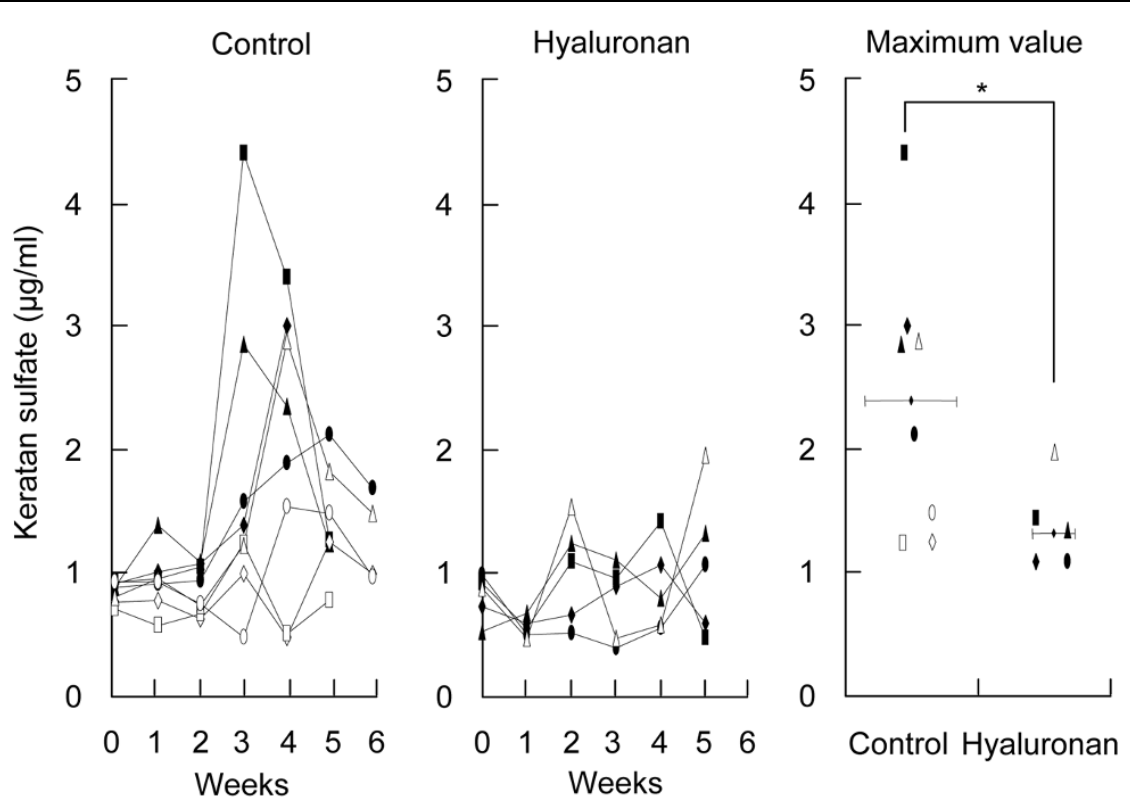

Serum concentration of keratan sulfate. Blood was collected every week at 0 to 5 weeks. Sequential serum concentrations of keratan sulfate are shown individually in the control group (left panel) and in the hyaluronan group (center panel). Maximum values of the concentrations are plotted, and the average values are shown in the right panel (control, $n=8$; injection of hyaluronan, $n=5$ ). ${ }^{\star \star} P<0.05$ by Mann-Whitney $\mathrm{U}$ test.

Figure 5

(a) (KS)
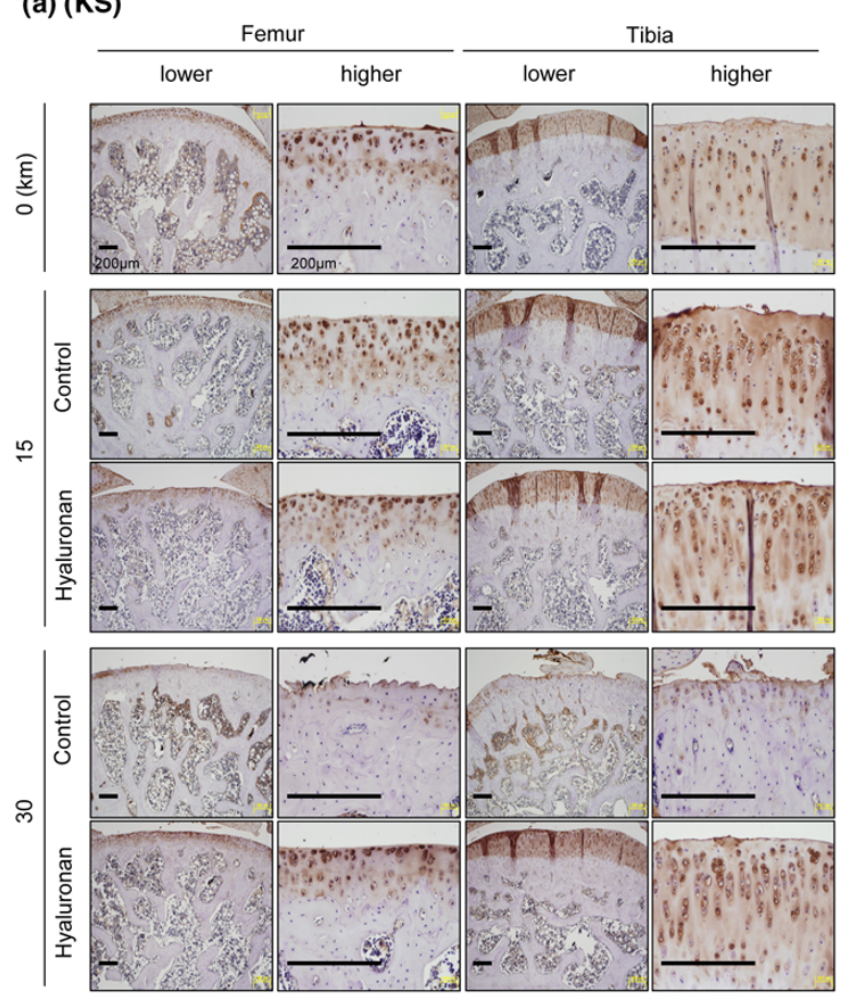

(b) (COL2)

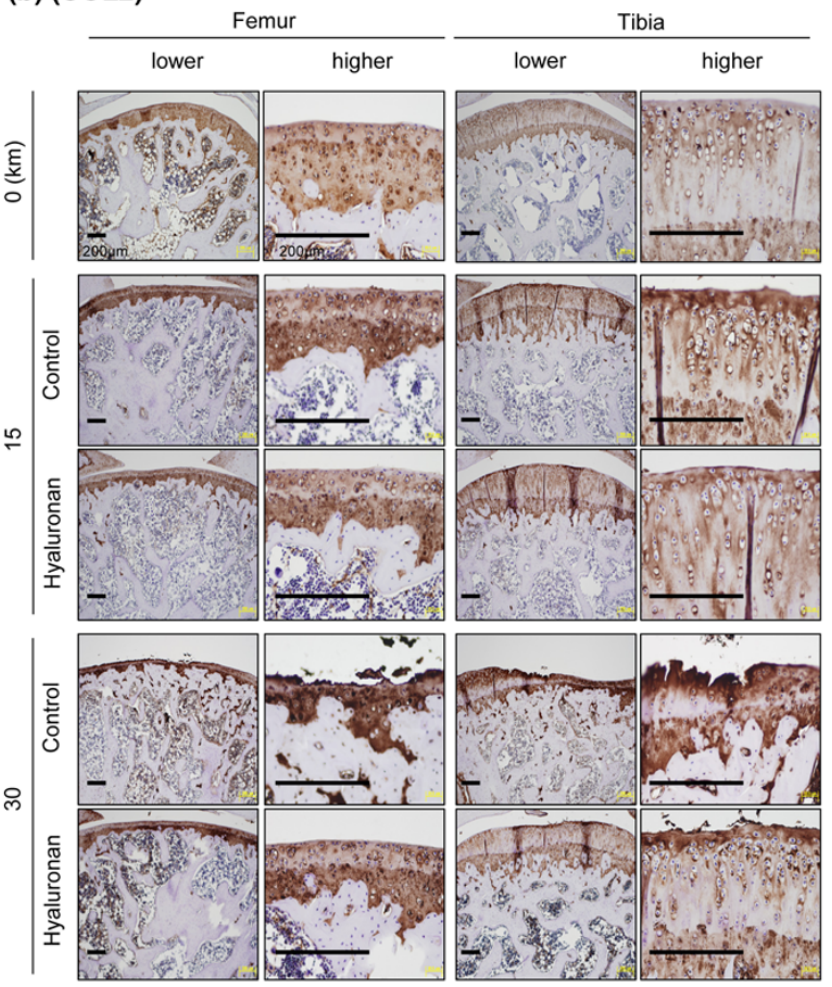

Immunohistochemical analyses. Representative histologies of the lateral femoral condyle and the lateral tibial plateau immunostained with (a) keratan sulfate (KS) and (b) type II collagen (COL2). Scale bar $=200 \mu \mathrm{m}$. 
such as cytokines, proteases, and enzyme inhibitors. In human subjects, serum keratan sulfate increased after exercise in healthy athletes [25] and in patients with early-stage osteoarthritis [12]. Furthermore, keratan sulfate was specifically distributed in the cartilage, cornea, and brain [8]. We therefore focused on serum keratan sulfate as an osteoarthritis marker in this rat model.

In the present study we demonstrated that serum keratan sulfate rapidly increased when Wistar rats ran approximately 15 to $20 \mathrm{~km}$. Interestingly, serum keratan sulfate rapidly decreased thereafter. According to our immunohistochemical analysis, keratan sulfate expression was still stable when rats ran $15 \mathrm{~km}$ and disappeared when rats ran 15 to $20 \mathrm{~km}$. We speculate a possible mechanism as follows. Daily strenuous exercise was extremely difficult for rats, and much of the resulting mechanical stress was absorbed by their joints. This caused transient joint cartilage degradation. Proteoglycan fragments, which are detached by mechanical stress to the cartilage, were ejected from the synovial cavity into the blood through the lymphatic system, and consequently serum keratan sulfate increased. Keratan sulfate in the affected cartilage also rapidly disappeared after keratan sulfate degraded once, and then serum keratan sulfate decreased rapidly.

Uebelhart and colleagues previously reported that serum keratan sulfate increased sharply 1 day after injection of chymopapain into the knee joint in rabbits [26]. The serum keratan sulfate also sharply decreased, and these changes were accompanied by depletion of proteoglycans evaluated by safranin-O stained histology. Although the cartilage matrix degradated in a much shorter period and keratan sulfate expression was not analyzed spatially or temporally in their model, their results correspond with our results in that serum keratan sulfate levels increased predictably following acute loss of proteoglycan.

For evaluation of keratan sulfate, we digested rat serum with Keratanase II and then measured the sum of monosulfate and disulfate disaccharides derived from keratan sulfate by HPLC. Rat serum may contain nonsulfate disaccharides, whose level may be affected by degeneration of the articular cartilage. In the present study, we cannot answer how the error of ignoring nonsulfated disaccharides could affect the measurement of the total keratan sulfate concentration in rat serum. We could, however, demonstrate that sulfated disaccharides derived from keratan sulfate in rat serum transiently increased in the early stages of osteoarthritis.

Our immunohistological analysis has shown that strenuous running led to damage of type II collagen in 6 weeks. We examined sequentially the serum concentration of $\mathrm{C} 2 \mathrm{C}$, specific for the destruction of type II collagen by MMP-1, MMP-8, and MMP-13. We could not, however, detect $\mathrm{C} 2 \mathrm{C}$ in rat serum in 6 weeks (data not shown).
Previous in vitro and in vivo studies indicate that exogenous hyaluronan can enhance proteoglycan synthesis and can prevent its release from the cell matrix $[16,27]$. Hyaluronan also suppresses the production and activity of proinflammatory mediators and proteases as well as altering the function of immune cells [17]. Intraarticular hyaluronan injection can reduce painful symptoms and improve general activities and joint mobility [28]. The mechanism may be that intraarticular hyaluronan injection causes less friction between articular cartilages, and improves joint comeback and reduces pain, thus providing good balance of the knee joint for running exercise.

Lammi and colleagues investigated the distribution of endogenous hyaluronan in full thickness defects of rat articular cartilage [6]. In normal articular cartilage, hyaluronan was stained mainly around the chondrocytes. During repair, strong hyaluronan staining was observed in loose mesenchymal tissue and in an area undergoing endochondral ossification. The high level of endogenous hyaluronan, however, could not induce the repair of osteochondral defect. Interestingly, remarkably strong staining for hyaluronan was demonstrated in areas that were simultaneously devoid of staining for keratan sulfate [6]. These results may show the possibility that the effect of endogenous hyaluronan is insufficient to repair the cartilage defect, losing keratan sulfate expression.

The present in vivo study demonstrated that intraarticular injection of hyaluronan suppressed progression of osteoarthritis. One of the mechanisms was to prevent release of keratan sulfate from the cartilage matrix. This could be monitored by the concentration of keratan sulfate in serum.

\section{Conclusion}

Osteoarthritic change could be obtained in a rat strenuous running model. Rat serum keratan sulfate was detected by HPLC and transiently increased along with depletion of keratan sulfate in cartilage tissue. Hyaluronan treatment suppressed development of osteoarthritis, and the effect was reflected by serum keratan sulfate.

\section{Competing interests}

The present work was supported by Seikagaku Corporation.

\section{Authors' contributions}

TT carried out the animal experiments, analyzed the results, and drafted the manuscript. TMu designed the initial plan. Y$\mathrm{JJ}, \mathrm{AN}$, and TMo assisted in the animal experiments. KM and HM examined the keratan sulfate concentration. IS conducted the experiments, participated in the evaluation, and completed the final manuscript. All authors read and approved the final manuscript.

\section{Acknowledgements}

The present study is supported in part by grants from the Japan Society for the Promotion of Science (19591752) and from the Center of Excellence Program for Frontier Research on Molecular Destruction and 
Reconstruction of Tooth and Bone in Tokyo Medical and Dental University to $\mathrm{TMu}$, and by a grant from the Japan Society for the Promotion of Science (18591657) to IS. Hyaluronan was distributed by Seikagaku Corp. (Tokyo, Japan).

\section{References}

1. Bendele AM: Animal models of osteoarthritis. J Musculoskelet Neuronal Interact 2001, 1:363-376.

2. van den Berg WB: Lessons from animal models of osteoarthritis. Curr Opin Rheumatol 2001, 13:452-456.

3. Pap G, Eberhardt R, Sturmer I, Machner A, Schwarzberg H, Roessner A, Neumann W: Development of osteoarthritis in the knee joints of Wistar rats after strenuous running exercise in a running wheel by intracranial self-stimulation. Pathol Res Pract 1998, 194:41-47.

4. Kujala UM, Kettunen J, Paananen $\mathrm{H}$, Aalto T, Battie MC, Impivaara $\mathrm{O}$, Videman T, Sarna S: Knee osteoarthritis in former runners, soccer players, weight lifters, and shooters. Arthritis Rheum 1995, 38:539-546.

5. Cymet TC, Sinkov V: Does long-distance running cause osteoarthritis? J Am Osteopath Assoc 2006, 106:342-345.

6. Lammi PE, Lammi MJ, Tammi RH, Helminen HJ, Espanha MM: Strong hyaluronan expression in the full-thickness rat articular cartilage repair tissue. Histochem Cell Bio/ 2001, 115:301-308.

7. Espanha MM, Lammi PE, Hyttinen MM, Lammi MJ, Helminen HJ: Extracellular matrix composition of full-thickness defect repair tissue is little influenced by exercise in rat articular cartilage. Connect Tissue Res 2001, 42:97-109.

8. Funderburgh JL: Keratan sulfate: structure, biosynthesis, and function. Glycobiology 2000, 10:951-958.

9. Campion GV, McCrae F, Schnitzer TJ, Lenz ME, Dieppe PA, Thonar EJ: Levels of keratan sulfate in the serum and synovial fluid of patients with osteoarthritis of the knee. Arthritis Rheum 1991, 34:1254-1259.

10. Thonar EJ, Lenz ME, Klintworth GK, Caterson B, Pachman LM, Glickman P, Katz R, Huff J, Kuettner KE: Quantification of keratan sulfate in blood as a marker of cartilage catabolism. Arthritis Rheum 1985, 28:1367-1376.

11. Tomatsu S, Okamura K, Maeda H, Taketani T, Castrillon SV, Gutierrez MA, Nishioka T, Fachel AA, Orii KO, Grubb JH, Cooper A, Thornley M, Wraith E, Barrera LA, Laybauer LS, Giugliani R, Schwartz IV, Frenking GS, Beck M, Kircher SG, Paschke E, Yamaguchi S, Ullrich K, Haskins M, Isogai K, Suzuki Y, Orii T, Kondo N, Creer M, Okuyama T, Tanaka A, Noguchi A: Keratan sulphate levels in mucopolysaccharidoses and mucolipidoses. J Inherit Metab Dis 2005, 28:187-202.

12. Wakitani S, Nawata M, Kawaguchi A, Okabe T, Takaoka K, Tsuchiya T, Nakaoka R, Masuda H, Miyazaki K: Serum keratan sulfate is a promising marker of early articular cartilage breakdown. Rheumatology (Oxford) 2007, 46:1652-1656.

13. Venn G, Mason RM: Absence of keratan sulphate from skeletal tissues of mouse and rat. Biochem J 1985, 228:443-450.

14. Ciombor DM, Lester G, Aaron RK, Neame P, Caterson B: Low frequency EMF regulates chondrocyte differentiation and expression of matrix proteins. J Orthop Res 2002, 20:40-50.

15. Divine JG, Zazulak BT, Hewett TE: Viscosupplementation for knee osteoarthritis: a systematic review. Clin Orthop Relat Res 2007, 455:113-122.

16. Goldberg VM, Buckwalter JA: Hyaluronans in the treatment of osteoarthritis of the knee: evidence for disease-modifying activity. Osteoarthritis Cartilage 2005, 13:216-224.

17. Moreland LW: Intra-articular hyaluronan (hyaluronic acid) and hylans for the treatment of osteoarthritis: mechanisms of action. Arthritis Res Ther 2003, 5:54-67.

18. Burghardt PR, Fulk LJ, Hand GA, Wilson MA: The effects of chronic treadmill and wheel running on behavior in rats. Brain Res 2004, 1019:84-96.

19. Reagan BF, Mclnerny VK, Treadwell BV, Zarins B, Mankin HJ: Irrigating solutions for arthroscopy. A metabolic study. J Bone Joint Surg Am 1983, 65:629-631.

20. Hulmes DJ, Marsden ME, Strachan RK, Harvey RE, Mclnnes N, Gardner DL: Intra-articular hyaluronate in experimental rabbit osteoarthritis can prevent changes in cartilage proteoglycan content. Osteoarthritis Cartilage 2004, 12:232-238.
21. Mankin HJ, Dorfman H, Lippiello L, Zarins A: Biochemical and metabolic abnormalities in articular cartilage from osteoarthritic human hips. II. Correlation of morphology with biochemical and metabolic data. J Bone Joint Surg Am 1971, 53:523-537.

22. Yokoyama A, Muneta $T$, Nimura $A$, Koga $H$, Mochizuki $T$, Hata $Y$, Sekiya I: FGF2 and dexamethasone increase the production of hyaluronan in two-dimensional culture of elastic cartilagederived cells: in vitro analyses and in vivo cartilage formation. Cell Tissue Res 2007, 329:469-478.

23. Koga H, Muneta T, Ju YJ, Nagase T, Nimura A, Mochizuki T, Ichinose S, von der Mark K, Sekiya I: Synovial stem cells are regionally specified according to local microenvironments after implantation for cartilage regeneration. Stem Cells 2007, 25:689-696.

24. Rousseau JC, Delmas PD: Biological markers in osteoarthritis. Nat Clin Pract Rheumatol 2007, 3:346-356.

25. Roos H, Dahlberg L, Hoerrner LA, Lark MW, Thonar EJ, Shinmei M, Lindqvist U, Lohmander LS: Markers of cartilage matrix metabolism in human joint fluid and serum: the effect of exercise. Osteoarthritis Cartilage 1995, 3:7-14.

26. Uebelhart $D$, Thonar EJ, Zhang J, Williams JM: Protective effect of exogenous chondroitin 4,6-sulfate in the acute degradation of articular cartilage in the rabbit. Osteoarthritis Cartilage 1998, 6(Suppl A):6-13.

27. Smith GN Jr, Myers SL, Brandt KD, Mickler EA: Effect of intraarticular hyaluronan injection in experimental canine osteoarthritis. Arthritis Rheum 1998, 41:976-985.

28. Gotoh S, Onaya J, Abe M, Miyazaki K, Hamai A, Horie K, Tokuyasu $\mathrm{K}$ : Effects of the molecular weight of hyaluronic acid and its action mechanisms on experimental joint pain in rats. Ann Rheum Dis 1993, 52:817-822. 